\title{
Psychometric Properties of the Chinese Version of the Cognitions About Body and Health Questionnaire
}

\author{
Shih-Cheng Liao iD ${ }^{1,2}$ \\ Wei-Lieh Huang ${ }^{2-4}$ \\ 'Department of Psychiatry, National \\ Taiwan University Hospital, Taipei, \\ Taiwan; ${ }^{2}$ Department of Psychiatry, \\ College of Medicine, National Taiwan \\ University, Taipei, Taiwan; ${ }^{3}$ Department \\ of Psychiatry, National Taiwan University \\ Hospital Yunlin Branch, Yunlin, Taiwan; \\ ${ }^{4}$ Graduate Institute of Clinical Medicine, \\ College of Medicine, National Taiwan \\ University, Taipei, Taiwan
}

Introduction: The Cognitions About Body and Health Questionnaire (CABAH) is a selfrating questionnaire measuring cognitions about health. The psychometric properties of its Chinese version and its performance on evaluating somatic symptom disorder (SSD) were examined in this study.

Methods: After translating the CABAH into Chinese, we collected data of the CABAH and another four questionnaires (Patient Health Questionnaire-15, Health Anxiety Questionnaire, Beck Depression Inventory-II, Beck Anxiety Inventory) in 208 SSD patients and 197 healthy individuals. We used exploratory factor analysis (EFA) to explore the structure of the Chinese CABAH. Internal consistency and criterion-related validity were analyzed. An independent $t$-test and receiver operating characteristic (ROC) curve analysis were used to evaluate the performance of the CABAH for evaluating SSD.

Results: Six factors were suggested by EFA. Five (bodily weakness, health habits, catastrophizing interpretation of specific bodily complaints, somatosensory amplification, catastrophizing interpretation of general bodily complaints) are conceptually associated with the original CABAH. The sixth factor (reverse, with three items) is different from the original construct. Cronbach's alpha for the CABAH was 0.885 . The CABAH score was moderately correlated with scores of the other four questionnaires. Scores for the whole CABAH and scores of the six factors were all significantly higher in SSD patients than in healthy individuals. The results of ROC curve analysis were as follows: area under the curve $=0.700$; suggested cutoff $=58 / 59$; Youden's $J=0.295$.

Conclusion: The reliability and validity of the Chinese CABAH were fair, although the three items in the reverse factor should be interpreted cautiously.

Keywords: Cognitions About Body and Health Questionnaire, psychometric properties, bodily weakness, somatic symptom disorder

\section{Introduction}

Somatoform disorders are defined in the Diagnostic and Statistical Manual of Mental Disorders, Fourth Edition (DSM-IV) for the individuals with medically unexplained physical symptoms. In the Diagnostic and Statistical Manual of Mental Disorders, Fifth Edition (DSM-5), the name of this diagnostic category was changed to somatic symptom and related disorders, in which the psychological features (such as catastrophizing cognitive style and health anxiety) are emphasized to replace the medically unexplained criteria. ${ }^{1,2}$ Somatic symptom disorder (SSD), the most common diagnosis of this category, has a criterion (criterion B) for the
Correspondence: Wei-Lieh Huang Department of Psychiatry, National Taiwan University Hospital Yunlin Branch Email weiliehhuang@gmail.com 
cognitive, affective or behavioral features of body and health. ${ }^{3}$ Although there have been questionnaires developed for measuring such psychological features (such as Somatic Symptom Disorder-B Criteria Scale, SSD-12) ${ }^{4}$ or questionnaires that examine associations with SSD criterion B (such as Health Anxiety Questionnaire, HAQ), 2,5 these tools do not focus on the cognitive domain about health. The cognitive aspect of the somatic symptoms has gained more and more attention in the current diagnostic system. For example, the catastrophizing cognitive style is listed as one feature of SSD, whereas excessive attention to the body is described in bodily distress disorder, a similar construct to SSD in the International Classification of Diseases 11th Revision. ${ }^{6}$ Several other cognitive features have been reported to be associated with somatic symptoms, including memory bias, the feature of expectation, and health attitude. ${ }^{7}$ Perceptual features, such as somatosensory amplification, are also associated with cognition. ${ }^{7}$ Therefore, a quantitative tool focusing on the cognitive domain should be helpful for clarifying the relations between the above features and the contemporary somatic diagnoses. To the best of our knowledge, the Cognitions About Body and Health Questionnaire (CABAH) is one of the most comprehensive tools for measuring cognition about health. ${ }^{8}$

The CABAH was developed by Rief et al; ${ }^{8}$ it has different versions with 31 and 39 items, and the 31-item version was originated from the previous 68 -item version. A four-point Likert scale (0-3) was used in the CABAH, with a higher value indicating higher severity. ${ }^{8}$ The 31 item CABAH includes catastrophizing interpretation of bodily complaints (14 items), autonomic sensations (4 items), bodily weakness (6 items), intolerance of bodily complaints (4 items) and health habits (3 items). The 39item version of the $\mathrm{CABAH}$ additionally incorporates the Somatosensory Amplification Scale (10 items, two overlaps with autonomic sensations) developed by Barsky et al. ${ }^{8,9}$ Two items in the CABAH were scored in reverse. The scoring principle is to sum the scores of all items; the scores of the different factors can be estimated separately. ${ }^{8,10}$ According to Rief et al.'s research, ${ }^{8}$ the internal consistency for the whole CABAH was 0.90 and $0.67-0.88$ for the different factors. The correlations between several factors and the Whiteley Index (WI, a measurement of hypochondriasis) score were 0.04-0.45. These psychometric properties indicate CABAH to be a useful tool, but there was no Chinese version of $\mathrm{CABAH}$ in the past.
The CABAH has been widely applied in the psychosomatic field. Comparing patients with somatoform disorder, patients with hypochondriasis had significantly higher CABAH scores. ${ }^{10}$ After receiving cognitive-behavioral therapy (CBT), CABAH scores of individuals with health anxiety significantly improved. ${ }^{11}$ For patients with hypochondriasis, responders to CBT had significantly lower CABAH scores than non-responders. ${ }^{12} \mathrm{CABAH}$ scores were associated with functioning and quality of life in SSD patients. ${ }^{13}$ Apart from the above findings, we are also interested in whether the cognitive feature in the diagnostic criteria of SSD can be presented via the CABAH score, and whether the CABAH can be used for screening, diagnosing or evaluating SSD. This issue can be explored by comparing CABAH scores in individuals with and without SSD. A similar approach has been adopted for investigating other psychosomatic questionnaires, such as the Patient Health Questionnaire-15 (PHQ-15), WI-7 and HAQ. ${ }^{5,14,15}$

There are two major aims in the present study. The first is to explore the psychometric properties of the Chinese version of the CABAH. The second is to investigate whether the CABAH is suitable for differentiating between SSD and healthy individuals and whether it can be applied to evaluate SSD.

\section{Methods}

\section{Translation of the CABAH}

We contacted the author of the CABAH to obtain permission for translating its 39-item version (which includes the Somatosensory Amplification Scale) into Chinese. ${ }^{8}$ The English version of the CABAH was initially translated into Chinese (forward-translation) by one author, then two authors discussed the translation and made necessary changes. Three psychosomatic patients were invited to read the questionnaire in order to limit any possible misunderstanding. We then requested a bi-lingual (English and Chinese) expert to perform a backward-translation (Chinese to English). When the two authors both considered that the meaning of sentences after backwardtranslation changed, we assumed that the sentences of forward-translation were not understood correctly; the Chinese sentences were revised under this circumstance. At the end of this process, we attained the Traditional Chinese version (based on the vocabulary in Taiwan) of the CABAH, which was used in the following procedure. This material originally appeared in English as Cognitive 
aspects of hypochondriasis and the somatizationsyndrome. Copyright (C) 1998 by American Psychological Association. Translated and reproduced with permission. American Psychological Association is not responsible for the quality or accuracy of this translation. This translation cannot be reproduced or distributed further without prior written permission.

\section{Procedure of the Psychometric Analysis}

The CABAH data from two studies were collected and analyzed. The Institutional Review Board of National Taiwan University Hospital approved the execution of this analysis (approval number: 201808047RINB). The aims of the two studies were both to investigate the psychological and biological features of SSD patients, some results of these studies have been published. ${ }^{13,16}$ All participants completed informed consent before gathering the data; this analysis was conducted in accordance with the declaration of Helsinki. Enrollment was from 2016 to 2020 at the National Taiwan University Hospital Yunlin Branch (NTUHYL). The inclusion criteria of the two studies are as follows: (1) patients with SSD, anxiety, or depression; (2) healthy individuals. The exclusion criteria of them are as follows: (1) age lower than 20 or higher than 70 years old; (2) having psychotic symptoms; (3) having difficulty completing questionnaires. To examine whether the CABAH is suitable for evaluating SSD, two populations were included in this analysis: SSD patients who visited psychiatric clinics at the NTUHYL and healthy individuals living in communities near the NTUHYL. Because there is not yet a structuralized tool for performing DSM-5-based diagnostic interview in Taiwan (such as The Structured Clinical Interview for DSM-5), we developed some semi-structured questions for diagnosing $\mathrm{SSD}$; the content has been described in our another study. ${ }^{17}$ We found 410 eligible subjects at first but five did not complete the questionnaire, leaving a final sample size of 405 for statistical analysis (208 SSD patients and 197 healthy individuals; mean age $44.16 \pm$ 12.42 years, 139 male). Their detailed demographic data are shown in Supplementary Table 1. All included subjects completed the $\mathrm{CABAH}$ and also another four questionnaires commonly used in the psychosomatic field in Taiwan: the PHQ-15, HAQ, Beck Depression Inventory-II (BDI-II) and Beck Anxiety Inventory (BAI). PHQ-15 and HAQ have been applied for screening SSD in a previous study. ${ }^{2}$ The data of all questionnaires from each subject were gathered in one single day.

\section{Other Questionnaires}

Scores of the PHQ-15, HAQ, BDI-II and BAI were gathered for examining the criterion-related validity (concurrent validity) of CABAH.

The PHQ-15, developed by Kroenke et al., ${ }^{18}$ measures the types and severity of somatic symptoms. It has 15 items; the score for each item is $0-2$ (higher score indicates more severe symptoms). The items can be separated into three major factors: cardiopulmonary, pain-fatigue and gastrointestinal. ${ }^{14}$ The internal consistency (Cronbach's alpha) of the Chinese PHQ-15 was 0.861.

The authors of the HAQ are Lucock and Morley. ${ }^{19}$ HAQ is a 21-item questionnaire aimed at measuring health anxiety and hypochondriacal ideation, with scoring on a four-point Likert scale (0-3). There were three major factors in the Chinese version of the HAQ: excessive worry about health and illness; the extent to which symptoms interfere with a person's life; and reassuranceseeking behavior. Its Cronbach's alpha was $0.943 .{ }^{5}$

The BDI-II was developed by Beck. ${ }^{20}$ It measures the level of depression, with coverage of cognitive/affective and somatic/vegetative dimensions. There are 21 items (score 0-3 for each) and Cronbach's alpha of the Chinese BDI-II was 0.94. ${ }^{21}$

The BAI was also developed by Beck. ${ }^{22}$ BAI emphasizes the somatic features of anxiety. It has 21 items, with scoring on a four-point Likert scale (0-3). Cronbach's alpha of the Chinese BAI was $0.95 .^{23}$

\section{Statistical Analysis}

We firstly used confirmatory factor analysis to examine the structure reported by Rief et al., ${ }^{8}$ but the model fit was not satisfying and did not obviously improve under minor modification (it was described in detail in Results). Therefore, exploratory factor analysis (EFA) was used to clarify the structure of the Chinese version of the CABAH. Principal component analysis with the Kaiser criterion was adopted for determining the number of factors to extract (the scree plot was shown in Supplementary Figure 1). We then used principal axis factoring with direct oblimin rotation to generate a pattern matrix. For comparison with the original CABAH, all items were classified into one factor according to the highest loadings (even when the highest loadings were lower than 0.4). We also performed item analysis for clarifying the features of each item. We then estimated internal consistency with Cronbach's alpha for the whole CABAH and for each 
factor; above $0.6-0.7$ and above 0.8 are usually considered as the acceptable and good values of Cronbach's alpha, respectively. ${ }^{24}$ With regard to criterion-related validity, we used Pearson's correlation analysis to calculate the association between the scores of the whole CABAH/six factors and four questionnaires (PHQ-15, HAQ, BDI-II, $\mathrm{BAI})$. Moreover, the difference in the scores of the whole CABAH/six factors between SSD patients and healthy individuals was compared using an independent $t$-test. Finally, receiver operating characteristic (ROC) curve analysis was adopted to examine whether the CABAH was suitable for diagnosing SSD; Youden's $\mathrm{J}$ (sensitivity + specificity -1 ) was calculated for determining the cutoff (the score with the highest Youden's J was considered as the optimal cutoff). Two-sided analysis with an alpha value of 0.05 was set for the $t$-test and correlation analysis. We used SPSS 25 and AMOS 25 (IBM, USA) for performing these analyses.

\section{Results}

\section{Confirmatory Factor Analysis According to the Original Structure}

The model fit statistics of confirmatory factor analysis according to the structure of the original CABAH were: $\mathrm{X}^{2}=1827.138, \quad \mathrm{X}^{2} / \mathrm{df}=2.667, \quad \mathrm{NFI}=0.646, \quad \mathrm{RFI}=0.597$, $\mathrm{IFI}=0.745, \mathrm{CFI}=0.740, \mathrm{TLI}=0.704$. These model fits were unsatisfactory, and EFA was warranted.

\section{Exploratory Factor Analysis}

The results of EFA revealed that there were six factors in the Chinese CABAH (Table 1). According to their features and similarity with the original $\mathrm{CABAH}$, we named them: bodily weakness (factor 1; items 1, 4, 5, 11, 14, 17, 23, 25, 30), health habits (factor 2; items 6, 9, 13, 18, 19, 26), catastrophizing interpretation of specific bodily complaints (factor 3; items 2, 10, 15, 20, 21, 24, 27, 28, 32), somatosensory amplification (factor 4; items 33, 34, 35, 36, 37, 38, 39), catastrophizing interpretation of general bodily complaints (factor 5; items 7, 8, 12, 16, 29) and reverse (factor 6; items 3, 22, 31). The detailed content of each factor and item (including a comparison of the structure of the Chinese and original CABAH versions) is shown in Supplementary Material.

\section{Internal Consistency}

Cronbach's alpha for the whole questionnaire (CABAH total) was good (0.885) and for factors $1-5$ it was $0.643-$
0.826, which is acceptable. However, Cronbach's alpha for factor 6 (reverse) was quite low $(-0.023)$. The results are shown in Table 2.

\section{Item Analysis}

Supplementary Table 2 gives the results of item analysis. In this step, we estimated the following values for all items: mean, variance, coefficient of skewness, correlation coefficient with CABAH total score and Cronbach's alpha of CABAH total after excluding this item. There was obvious skewness for item 3. All items except item 3 and item 22 were significantly correlated with $\mathrm{CABAH}$ total score. With regard to Cronbach's alpha of CABAH total after excluding an item, several items were related with elevating internal consistency (items 3, 19, 22, 31); these items were highly overlapping with the factor 6 (reverse).

\section{Criterion-Related Validity}

The correlations between the scores of CABAH total, the six factors and other questionnaires are shown in Table 3. The score of CABAH total was moderately correlated with the scores of the four questionnaires ( $r=0.416-0.535)$. Among the six factors, factor 1 (bodily weakness) score revealed the highest correlations with the four questionnaires ( $r=0.591-0.637$ ) scores. Factor 3 (catastrophizing interpretation of specific bodily complaints), factor 4 (somatosensory amplification) and factor 5 (catastrophizing interpretation of general bodily complaints) scores were significantly correlated with the four questionnaires ( $r=0.2-0.4)$ scores. Factor 2 (health habits) score was only significantly correlated with the HAQ score $(r=$ 0.230). Although factor 6 (reverse) score was significantly correlated with the HAQ and BAI scores, the correlation coefficients were low (0.106 and 0.124 , respectively).

\section{Comparison of CABAH Scores in SSD Patients and Healthy Individuals}

Table 4 and Supplementary Table 3 show the results from the comparison of CABAH total/six factors and all item scores, respectively. SSD patients showed significantly higher scores for CABAH total and all six factors than healthy individuals, especially factor 1 (bodily weakness, $t=12.595$ ). For most items, SSD patients had significantly higher scores, but there were exceptions: the scores on items 19 and 33 were significantly higher in healthy individuals; 
Table I Exploratory Factor Analysis of the Chinese Version of CABAH

\begin{tabular}{|c|c|c|c|c|c|c|}
\hline & Factor I & Factor 2 & Factor 3 & Factor 4 & Factor 5 & Factor 6 \\
\hline \multicolumn{7}{|c|}{ Factor I (bodily weakness) } \\
\hline CABAHQ25 & 0.643 & -0.050 & 0.041 & 0.183 & 0.035 & 0.046 \\
\hline CABAHQII & 0.592 & -0.083 & 0.015 & 0.120 & 0.189 & -0.048 \\
\hline CABAHQI4 & 0.561 & -0.067 & 0.151 & 0.021 & 0.110 & 0.072 \\
\hline CABAHQOI & 0.549 & 0.085 & -0.065 & 0.192 & 0.129 & -0.182 \\
\hline CABAHQI7 & 0.545 & 0.127 & 0.286 & -0.019 & -0.207 & 0.077 \\
\hline CABAHQ04 & 0.423 & -0.001 & 0.107 & 0.067 & 0.263 & 0.114 \\
\hline CABAHQ05 & 0.376 & 0.344 & -0.049 & 0.021 & 0.291 & -0.040 \\
\hline CABAHQ30 & 0.318 & -0.101 & 0.302 & 0.262 & 0.062 & 0.055 \\
\hline CABAHQ23 & 0.264 & -0.201 & 0.155 & 0.210 & -0.092 & 0.163 \\
\hline \multicolumn{7}{|c|}{ Factor 2 (health habits) } \\
\hline CABAHQI9 & -0.108 & 0.655 & -0.009 & 0.043 & -0.165 & 0.198 \\
\hline CABAHQI3 & -0.082 & 0.584 & 0.082 & 0.136 & 0.141 & -0.108 \\
\hline CABAHQ09 & 0.079 & 0.443 & 0.002 & 0.101 & 0.341 & -0.234 \\
\hline CABAHQ26 & 0.036 & 0.416 & 0.054 & 0.073 & 0.094 & 0.034 \\
\hline CABAHQ06 & 0.169 & 0.322 & 0.181 & 0.001 & 0.303 & -0.307 \\
\hline CABAHQI8 & 0.076 & 0.258 & 0.243 & 0.119 & 0.213 & -0.156 \\
\hline \multicolumn{7}{|c|}{$\begin{array}{l}\text { Factor } 3 \text { (catastrophizing interpretation of bodily complaints, specific } \\
\text { diseases) }\end{array}$} \\
\hline CABAHQIO & -0.142 & 0.020 & 0.590 & 0.031 & 0.211 & -0.181 \\
\hline CABAHQI5 & 0.209 & 0.012 & 0.533 & -0.014 & 0.022 & -0.079 \\
\hline CABAHQ02 & 0.135 & -0.007 & 0.525 & 0.020 & -0.017 & -0.170 \\
\hline CABAHQ27 & 0.033 & 0.007 & 0.510 & -0.060 & 0.244 & -0.060 \\
\hline CABAHQ28 & 0.235 & -0.035 & 0.509 & 0.134 & -0.132 & 0.052 \\
\hline CABAHQ32 & -0.097 & 0.009 & 0.460 & 0.048 & -0.073 & 0.066 \\
\hline CABAHQ2I & 0.239 & 0.254 & 0.363 & -0.022 & -0.200 & -0.036 \\
\hline CABAHQ24 & 0.030 & 0.122 & 0.354 & 0.093 & 0.055 & 0.153 \\
\hline CABAHQ20 & 0.107 & 0.120 & 0.332 & 0.078 & 0.254 & 0.088 \\
\hline \multicolumn{7}{|c|}{ Factor 4 (somatosensory amplification) } \\
\hline CABAHQ35 & 0.045 & 0.245 & 0.062 & 0.589 & -0.038 & 0.035 \\
\hline CABAHQ38 & 0.028 & 0.017 & 0.205 & $0.57 \mid$ & -0.209 & -0.196 \\
\hline CABAHQ36 & 0.130 & -0.069 & 0.019 & 0.548 & 0.089 & 0.025 \\
\hline CABAHQ39 & 0.110 & 0.036 & 0.091 & 0.477 & -0.090 & -0.178 \\
\hline CABAHQ34 & 0.071 & 0.141 & -0.183 & $0.45 I$ & 0.210 & 0.117 \\
\hline CABAHQ37 & 0.131 & -0.018 & 0.084 & 0.427 & 0.058 & -0.013 \\
\hline CABAHQ33 & -0.178 & 0.078 & -0.122 & 0.348 & 0.094 & 0.056 \\
\hline \multicolumn{7}{|c|}{ Factor 5 (catastrophizing interpretation of bodily complaints, general) } \\
\hline CABAHQ08 & 0.057 & 0.010 & 0.072 & 0.134 & 0.597 & 0.014 \\
\hline CABAHQ07 & 0.159 & 0.032 & 0.017 & -0.052 & 0.548 & 0.047 \\
\hline CABAHQI2 & -0.068 & 0.190 & 0.012 & 0.061 & 0.493 & 0.011 \\
\hline CABAHQI6 & -0.027 & 0.056 & 0.258 & 0.237 & 0.335 & 0.005 \\
\hline CABAHQ29 & 0.066 & -0.180 & 0.125 & 0.217 & 0.226 & 0.125 \\
\hline \multicolumn{7}{|l|}{ Factor 6 (reverse) } \\
\hline CABAHQ3I & 0.303 & -0.141 & -0.154 & 0.058 & -0.046 & -0.316 \\
\hline CABAHQ22 & -0.153 & -0.033 & 0.018 & 0.059 & 0.024 & -0.296 \\
\hline CABAHQ03 & -0.050 & -0.022 & -0.051 & 0.037 & 0.054 & 0.251 \\
\hline
\end{tabular}

Abbreviation: $\mathrm{CABAH}$, Cognitions About Body and Health Questionnaire. 
Table 2 Internal Consistency of the Chinese Version of CABAH, Including the Whole Questionnaire and 6 Factors

\begin{tabular}{|l|l|}
\hline & $\begin{array}{l}\text { Internal Consistency } \\
\text { (Cronbach's } \boldsymbol{\alpha} \text { ) }\end{array}$ \\
\hline CABAH total & 0.885 \\
Factor I (bodily weakness) & 0.826 \\
Factor 2 (health habits) & 0.741 \\
Factor 3 (catastrophizing interpretation of & 0.774 \\
bodily complaints, specific diseases) & \\
Factor 4 (somatosensory amplification) & 0.728 \\
Factor 5 (catastrophizing interpretation of & 0.643 \\
bodily complaints, general) & \\
Factor 6 (reverse) & -0.023 \\
\hline
\end{tabular}

Abbreviation: $\mathrm{CABAH}$, Cognitions About Body and Health Questionnaire.

and the scores on items $3,12,13,16,18,21,22,32$ and 35 did not reveal the significant inter-group difference.

\section{ROC Curve Analysis}

The results of the ROC curve analysis are shown in Figure 1 . The area under the curve was 0.700 , which is acceptable, and the suggested cutoff was 58/59; however, Youden's $J$ equaled 0.295 (sensitivity 0.615 , specificity

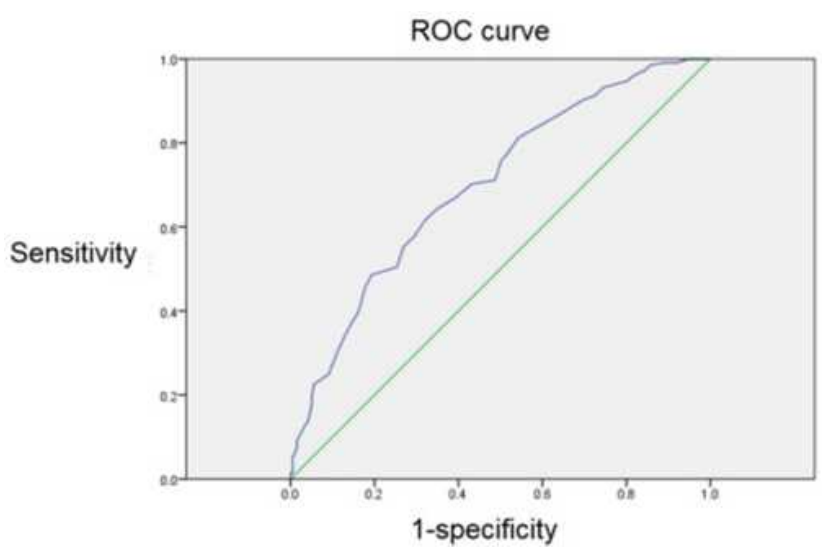

Figure I ROC curve analysis for the Chinese version of CABAH on detecting SSD. Area under curve $=0.700$, suggested cutoff $58 / 59$, Youden's $J=0.295$.

Abbreviations: ROC, receiver operating characteristic; CABAH, Cognitions About Body and Health Questionnaire; SSD, somatic symptom disorder.

0.680), which means that using the CABAH alone for diagnosing SSD is unsatisfactory.

\section{Discussion}

Compared with the structure of the original $\mathrm{CABAH}$, the Chinese version of CABAH revealed high similarity in the following three factors: bodily weakness, health habits and

Table 3 Criterion-Related Validity of the Chinese Version of CABAH

\begin{tabular}{|l|l|l|l|l|l|l|l|l|l|l|}
\hline & Factor I & Factor 2 & Factor 3 & Factor 4 & Factor 5 & Factor 6 & PHQ-I 5 & HAQ & BDI-II & BAI \\
\hline CABAH total & $0.815^{* * *}$ & $0.661^{* * *}$ & $0.772^{* * *}$ & $0.736^{* * *}$ & $0.691^{* * *}$ & $0.103^{*}$ & $0.442^{* * *}$ & $0.535^{* * *}$ & $0.416^{* * *}$ & $0.493^{* * *}$ \\
Factor I & $\mathrm{I}$ & $0.321^{* * *}$ & $0.559^{* * *}$ & $0.491^{* * *}$ & $0.457^{* * *}$ & 0.012 & $0.606^{* * *}$ & $0.61 I^{* * *}$ & $0.59 I^{* * *}$ & $0.637^{* * *}$ \\
Factor 2 & & $\mathrm{I}$ & $0.437^{* * *}$ & $0.417^{* * *}$ & $0.474^{* * *}$ & -0.054 & 0.028 & $0.230^{* * *}$ & -0.004 & $0.08 \mathrm{I}$ \\
Factor 3 & & & $\mathrm{I}$ & $0.416^{* * *}$ & $0.392^{* * *}$ & -0.054 & $0.274^{* * *}$ & $0.295^{* * *}$ & $0.262^{* * *}$ & $0.314^{* * *}$ \\
Factor 4 & & & & $\mathrm{I}$ & $0.455^{* * *}$ & 0.029 & $0.299^{* * *}$ & $0.379 * * *$ & $0.279^{* * *}$ & $0.325^{* * *}$ \\
Factor 5 & & & & & $\mathrm{I}$ & 0.028 & $0.257^{* * *}$ & $0.359^{* * *}$ & $0.256^{* * *}$ & $0.293^{* * *}$ \\
Factor 6 & & & & & & 1 & $0.08 \mathrm{I}$ & $0.106^{*}$ & 0.056 & $0.124^{*}$ \\
\hline
\end{tabular}

Notes: ${ }^{*} p<0.05, * * * p<0.001$.

Abbreviations: CABAH, Cognitions About Body and Health Questionnaire; PHQ-I5, Patient Health Questionnaire; HAQ, Health Anxiety Questionnaire; BDI-II, Beck Depression Inventory-II; BAI, Beck Anxiety Inventory.

Table 4 Comparison of the Chinese Version of CABAH Scores in Patients with SSD and Healthy Individuals

\begin{tabular}{|c|c|c|c|c|c|c|}
\hline & \multicolumn{2}{|c|}{ SSD $(n=208)$} & \multicolumn{2}{|c|}{ Healthy $(n=197)$} & \multicolumn{2}{|c|}{ Statistics } \\
\hline & Mean & SD & Mean & SD & $\mathbf{t}$ & $p$ value \\
\hline CABAH total & 62.51 & 13.06 & 52.25 & 14.03 & 7.624 & $<0.001 * * *$ \\
\hline Factor I (bodily weakness) & 15.09 & 4.60 & 9.47 & 4.34 & 12.595 & $<0.00 I^{* * *}$ \\
\hline Factor 2 (health habits) & 10.25 & 3.16 & 9.57 & 3.46 & 2.069 & $0.039 *$ \\
\hline Factor 3 (catastrophizing interpretation of bodily complaints, specific diseases) & 9.61 & 4.20 & 8.20 & 4.30 & 3.323 & $0.001 * *$ \\
\hline Factor 4 (somatosensory amplification) & 11.89 & 3.69 & 10.50 & 3.45 & 3.915 & $<0.001^{* * *}$ \\
\hline Factor 5 (catastrophizing interpretation of bodily complaints, general) & 10.50 & 2.46 & 9.65 & 2.63 & 3.367 & $0.00 I^{* *}$ \\
\hline Factor 6 (reverse) & 5.17 & 2.04 & 4.81 & 1.20 & 2.189 & $0.029 *$ \\
\hline
\end{tabular}

Notes: ${ }^{*} \mathrm{p}<0.05,{ }^{*} \mathrm{p}<0.01, * * * \mathrm{p}<0.001$.

Abbreviations: CABAH, Cognitions About Body and Health Questionnaire; SSD, somatic symptom disorder. 
somatosensory amplification. ${ }^{8}$ The factor of catastrophizing interpretation of bodily complaints, which has the most items in the original $\mathrm{CABAH}$, can be separated into two factors in our sample: catastrophizing about specific and general bodily complaints. The other two factors in the original $\mathrm{CABAH}$, autonomic sensations and intolerance of bodily complaints, are not so distinct in our study. In this analysis, we found another factor consisting of three items, two of which were reversely scored and showed negative loadings in the pattern matrix. We have carefully checked the coding and confirm that the negative loadings did not originate from the coding error. We named this factor "reverse"; its three items showed problems also in other analyses besides EFA. Moreover, low loadings $(<0.3)$ were found in several items, such as in item 23 (I can sometimes hear my pulse or my heartbeat throbbing in my ear) of factor 1 (bodily weakness), in item 18 (If I do not observe my body often, I could become seriously ill without noticing it) of factor 2 (health habits) and in item 29 (I hate to be too hot or too cold) of factor 5 (catastrophizing interpretation of general bodily complaints). These items had relatively low convergent validity and did not belong to the similar factors of the original $\mathrm{CABAH}$; therefore, it is rational that they were less connected with other items of the same factors. ${ }^{8}$ Items with low factor loadings are sometimes deleted during the development of questionnaires; however, because the full version of CABAH was used in most published articles, we chose to preserve the full $\mathrm{CABAH}$ in these analyses. If performing an additional confirmatory factor analysis after excluding items with low factor loadings, the model fit would improve $\left(\mathrm{X}^{2}=278.353, \quad \mathrm{X}^{2} / \mathrm{df}=2.554, \quad \mathrm{NFI}=0.848, \quad \mathrm{RFI}=0.786\right.$, $\mathrm{IFI}=0.901, \mathrm{CFI}=0.899, \mathrm{TLI}=0.858$ ).

The internal consistency of 0.855 for the whole CABAH is a good level and similar to that of the original version (0.9). ${ }^{8}$ Separating the different factors, factor 1 (bodily weakness) has the highest internal consistency of 0.826 , followed by $0.6-0.8$ for factors $2-5$; these values are similar to those for the original $\mathrm{CABAH} .{ }^{8}$ However, the internal consistency of factor 6 (reverse) was quite low $(-0.23)$; this may be explained by the one positive (item 3 ) and two negative loadings (items 22, 31). Removing these three items from analysis would lead to higher internal consistency; these items also showed lower correlations with $\mathrm{CABAH}$ total scores or high levels of skewness. Reverse item performance in item analysis is often not as good as other items in psychometric studies, so the results are not surprising. ${ }^{25}$ With regard to item 3, we suppose the finding to be associated with the translated sentence. The original content of item 3 is "I am healthy when I do not have any bodily sensations"; this means that the healthy cognition exists "only when" the subjects did not feel any discomfort; however, we translated this sentence directly and did not additionally emphasize the concept "only when", so some subjects might have misunderstood this statement. In summary, items 3, 22 and 31 of the Chinese CABAH should be used and interpreted cautiously.

The CABAH total scores were moderately correlated with the scores of four commonly used questionnaires in the psychosomatic field. This implies that the constructs in the CABAH cannot be replaced by other quantitative measurements. Due to the coexisting tendency of somatic distress, depression, anxiety and hypochondriacal ideation, it is rational that the correlations were not very low. ${ }^{5,15}$ Factor 1 (bodily weakness) scores showed the highest correlations with all four questionnaires scores. A similar finding was reported in the original $\mathrm{CABAH}$; the bodily weakness factor score showed the highest correlation with the WI score than the other factors. ${ }^{8}$ This implies that "weakness" may be an overlapping concept with both somatic distress and negative emotions. On the other hand, factor 2 (health habits) seemed quite different from all the constructs of the other questionnaires; only the HAQ score revealed a significant (but low) correlation with it. An explanation for this is that health habits are features that transit from cognition to behavior, which are not measured in the four questionnaires. Whether a measurement of illness behavior (such as the Scale for the Assessment of Illness Behavior) shows a higher association with the health habits factor warrants further exploration. $^{26}$

Scores of the CABAH total and of the six factors were significantly higher in SSD patients than in healthy individuals, which is quite rational. Score of factor 6 (reverse) was also significantly higher in SSD patients; this phenomenon indicates that although the reverse items had a different presentation approach, not all subjects misunderstood their meanings. The inter-group difference was highest for factor 1 (bodily weakness), implying that the cognition about "weakness" is the clearest feature of SSD patients in Taiwan; thus, "weakness" questions may be helpful for the clinical purpose of SSD screening. "Neurasthenia" is still a commonly used term in Taiwan and in China; its original meaning is about the weakness. ${ }^{27}$ Therefore, the emphasis on bodily weakness may have a cultural meaning, especially in Chinese society. 
Besides, the cognition about weakness may be considered as an extended criterion of SSD or similar diagnoses. From ROC curve analysis, the low Youden's $J$ indicates that the sensitivity and specificity when applying CABAH alone for diagnosing SSD are not satisfactory. It is rational because only part of the SSD criteria (the "cognitive" criterion B1) can be assessed by CABAH. Some studies reported that combining different questionnaires (such as one for SSD criterion A and others for criterion B) may generate better performance in ROC curve analysis., 2,28,29 We also considered this approach and found when combining the scores of CABAH, PHQ-15 and HAQ, the area under the curve increased from 0.700 to 0.859 (Supplementary Figure 2).

Several limitations of this study need to be discussed. First, we did not collect data on test-retest reliability, therefore we cannot establish whether the Chinese CABAH is temporally stable. Second, we did not include data of non-SSD patients in the psychiatric clinic or psychosomatic patients in consultation-liaison situations, therefore, the suitability of applying the Chinese CABAH for these populations is not clear.

In sum, there are several implications of the present study. First, the structures of CABAH may be different in Taiwan and in Europe. Among the dimensions of somatic symptoms, PHQ-15, a questionnaire measuring the level of somatic distress, was found to have distinct structures in Chinese and European samples. ${ }^{30}$ Second, using only CABAH is not suitable for diagnosing SSD; the questionnaire SSD-12 should be the better option for this purpose. ${ }^{31}$ Different from the previous construct somatoform disorders, the concept "medically unexplained" has been removed in SSD; therefore, SSD may co-exist in patients with medical comorbidity. ${ }^{32,33}$ SSD-12 has been found to effectively diagnose SSD in a population with medical comorbidity. ${ }^{31}$ Third, though diagnosing SSD is not the strength of $\mathrm{CABAH}$, its abundant cognitive measurements may still have clinical values. A study revealed that SSD had high complexity and was more complex than some other diseases, such as multiple sclerosis and rheumatoid arthritis. ${ }^{34}$ It indicates further classification or detailed evaluation of SSD to be needed; CABAH can be an option for these aims. Another study showed that when patients with somatic symptom and related disorders had comorbid depression, they had poor neurocognitive performance; it may explain why some patients easily dropped out from $\mathrm{CBT}^{35}{ }^{35}$ Besides, the different diagnoses in somatic symptoms and related disorders may have distinct neurocognitive performance. For example, patients with conversion disorder were found to have relatively poor processing speed. ${ }^{36}$ The above features may be explored by using CABAH.

This study is a first attempt at clarifying the psychometric features of the Chinese version of the CABAH. Besides providing information on the structure of the Chinese CABAH and the suitability of items, we also investigated the possibility of applying the CABAH to the evaluation of SSD. In general, the Chinese CABAH has fair reliability and validity, although three items (items 3, 22, 31) in factor 6 (reverse) should be used and interpreted cautiously. We expect that the CABAH can be used more extensively in Chinese society; apart from clinical purposes, it may be beneficial for showing the potential cultural difference between Eastern and Western populations.

\section{Data Accessibility}

Data of this study are not shared.

\section{Acknowledgments}

We thank Professor Winfried Rief for providing us permission to translate the Cognitions About Body and Health Questionnaire into the Chinese version. We also thank Professor Arthur Joseph Barsky III for providing us permission to translate the Somatosensory Amplification Scale into the Chinese version.

\section{Author Contributions}

All authors made a significant contribution to the work reported, whether that is in the conception, study design, execution, acquisition of data, analysis and interpretation, or in all these areas; took part in drafting, revising or critically reviewing the article; gave final approval of the version to be published; have agreed on the journal to which the article has been submitted; and agree to be accountable for all aspects of the work.

\section{Funding}

This work was supported by the National Taiwan University Hospital Yunlin Branch (grant numbers NTUHYL105.X002, NTUHYL108.X001).

\section{Disclosure}

The authors report no conflicts of interest in this work. 


\section{References}

1. American Psychiatric Association. Diagnostic and Statistical Manual of Mental Disorders. 5th ed. Arlington, VA: American Psychiatric Association; 2013.

2. Huang WL, Chen IM, Chang FC, Liao SC. Somatic symptom disorder and undifferentiated somatoform disorder, which is broader? Response to "Clinical value of DSM IV and DSM 5 criteria for diagnosing the most prevalent somatoform disorders in patients with medically unexplained physical symptoms (MUPS)". $J \quad$ Psychosom Res. 2016;89:114-115. doi:10.1016/j. jpsychores.2016.07.014

3. Rief W, Martin A. How to use the new DSM-5 somatic symptom disorder diagnosis in research and practice: a critical evaluation and a proposal for modifications. Annu Rev Clin Psychol. 2014;10: 339-367. doi:10.1146/annurev-clinpsy-032813-153745

4. Toussaint A, Murray AM, Voigt K, et al. Development and validation of the somatic symptom disorder-B Criteria Scale (SSD-12). Psychosom Med. 2016;78(1):5-12. doi:10.1097/PSY.00000000000 00240

5. Chen IM, Huang WL, Lee MB, Liao SC. Chinese Version of Health Anxiety Questionnaire: psychometric properties and its association with DSM-IV/DSM-5 sytems. Formosan J Med. 2019;23:21-30.

6. Gureje O, Reed GM. Bodily distress disorder in ICD-11: problems and prospects. World Psychiatry. 2016;15(3):291-292. doi:10.1002/ wps. 20353

7. Rief W, Broadbent E. Explaining medically unexplained symptoms-models and mechanisms. Clin Psychol Rev. 2007;27 (7):821-841. doi:10.1016/j.cpr.2007.07.005

8. Rief W, Hiller W, Margraf J. Cognitive aspects of hypochondriasis and the somatization syndrome. J Abnorm Psychol. 1998;107 (4):587-595. doi:10.1037/0021-843X.107.4.587

9. Barsky AJ, Wyshak G, Klerman GL. The somatosensory amplification scale and its relationship to hypochondriasis. J Psychiatr Res. 1990;24(4):323-334. doi:10.1016/0022-3956(90)90004-A

10. Hiller W, Rief W, Fichter MM. Dimensional and categorical approaches to hypochondriasis. Psychol Med. 2002;32(4):707-718. doi:10.1017/S0033291702005524

11. Newby JM, Mahoney AEJ, Mason EC, Smith J, Uppal S, Andrews G. Pilot trial of a therapist-supported internet-delivered cognitive behavioural therapy program for health anxiety. Int Interventions. 2016;6:71-79. doi:10.1016/j.invent.2016.09.007

12. Hiller W, Leibbrand R, Rief W, Fichter MM. Predictors of course and outcome in hypochondriasis after cognitive-behavioral treatment. Psychother Psychosom. 2002;71(6):318-325. doi:10.1159/000065 990

13. Liao SC, Ma HM, Lin YL, Huang WL. Functioning and quality of life in patients with somatic symptom disorder: the association with comorbid depression. Compr Psychiatry. 2019;90:88-94. doi:10.10 16/j.comppsych.2019.02.004

14. Liao SC, Huang WL, Ma HM, et al. The relation between the patient health questionnaire-15 and DSM somatic diagnoses. $B M C$ Psychiatry. 2016;16(1):351. doi:10.1186/s12888-016-1068-2

15. Tu CY, Liao SC, Liu CY, et al. Application of the Chinese Version of the Whiteley Index-7 for Detecting DSM-5 Somatic symptom and related disorders. Psychosomatics. 2016;57(3):283-291. doi:10.1016/ j.psym.2015.12.010

16. Huang WL, Liao SC. Subgrouping somatic symptom disorder: an analysis based on the diagnostic criteria for psychosomatic research. Psychother Psychosom. 2018;87(5):316-318. doi:10.1159/000492 227

17. Tu CY, Liu WS, Chen YF, Huang WL. Is severity a clinically meaningful specifier of somatic symptom disorder? J Psychosom Res. 2020;133:110108. doi:10.1016/j.jpsychores.2020.110108
18. Kroenke K, Spitzer RL, Williams JB. The PHQ-15: validity of a new measure for evaluating the severity of somatic symptoms. Psychosom Med. 2002;64(2):258-266. doi:10.1097/00006842-200203000-00008

19. Lucock MP, Morley S. The health anxiety questionnaire. Br J Health Psychol. 1996;1(2):137-150. doi:10.1111/j.2044-8287.1996.tb00498.x

20. Beck AT, Steer RA, Ball R, Ranieri W. Comparison of Beck Depression Inventories-IA and -II in psychiatric outpatients. $J$ Pers Assess. 1996;67(3):588-597. doi:10.1207/s15327752jpa6703_13

21. Lu ML, Che HH, Chang SW, Shen WW. Reliability and validity of the Chinese version of the Beck Depression Inventory-II. Taiwanese $J$ Psychiatry. 2002;16(4):301-310.

22. Beck AT, Epstein N, Brown G, Steer RA. An inventory for measuring clinical anxiety: psychometric properties. J Consult Clin Psychol. 1988;56(6):893-897. doi:10.1037/0022-006X.56.6.893

23. Che HH, Lu ML, Chen HC, Chang SW, Li YC. Validation of the Chinese Version of the Beck Anxiety Inventory. Formosan J Med. 2006;4:447-454.

24. Ursachi G, Horodnic IA, Zait A. How reliable are measurement scales? External factors with indirect influence on reliability estimators. Procedia Economics Finance. 2015;20:679-686. doi:10. 1016/S2212-5671(15)00123-9

25. Swain SD, Weathers D, Niedrich RW. Assessing three sources of misresponse to reversed Likert items. J Marketing Res. 2008;45 (1):116-131. doi:10.1509/jmkr.45.1.116

26. Rief W, Ihle D, Pilger F. A new approach to assess illness behaviour. J Psychosom Res. 2003;54(5):405-414. doi:10.1016/S0022-3999(02) 00401-4

27. Schwartz PY. Why is neurasthenia important in Asian cultures? Western J Med. 2002;176(4):257-258.

28. Laferton JAC, Stenzel NM, Rief W, Klaus K, Brahler E, Mewes R. Screening for DSM-5 Somatic Symptom Disorder: diagnostic Accuracy of Self-Report Measures Within a Population Sample. Psychosom Med. 2017;79(9):974-981. doi:10.1097/PSY.0000000000000530

29. Toussaint A, Husing P, Kohlmann S, Lowe B. Detecting DSM-5 somatic symptom disorder: criterion validity of the Patient Health Questionnaire-15 (PHQ-15) and the somatic symptom scale-8 (SSS-8) in combination with the Somatic Symptom Disorder-B Criteria Scale (SSD-12). Psychol Med. 2020;50 (2):324-333. doi:10.1017/S003329171900014X

30. Leonhart R, de Vroege L, Zhang L, et al. Comparison of the factor structure of the patient health questionnaire for somatic symptoms (PHQ-15) in Germany, the Netherlands, and China. A Transcultural Structural Equation Modeling (SEM) Study. Front Psychiatry. 2018;9:240. doi:10.3389/fpsyt.2018.00240

31. Kop WJ, Toussaint A, Mols F, Lowe B. Somatic symptom disorder in the general population: associations with medical status and health care utilization using the SSD-12. Gen Hosp Psychiatry. 2019;56:36-41. doi:10.1016/j.genhosppsych.2018.10.004

32. van Eck van der Sluijs J, Ten Have M, Rijnders C, van Marwijk H, de Graaf R, van der Feltz-cornelis C. Medically unexplained and explained physical symptoms in the general population: association with prevalent and incident mental disorders. PLoS One. 2015;10(4): e0123274. doi:10.1371/journal.pone.0123274

33. van Eck van der Sluijs JF, Ten Have M, de Graaf R, Rijnders CAT, van Marwijk HWJ, van der Feltz-cornelis CM. Predictors of persistent medically unexplained physical symptoms: findings from a general population study. Front Psychiatry. 2018;9:613. doi:10. 3389/fpsyt.2018.00613

34. van Eck van der Sluijs JF, de Vroege L, van Manen AS, Rijnders CAT, van der Feltz-cornelis CM. Complexity Assessed by the INTERMED in patients with somatic symptom disorder visiting a specialized outpatient mental health care setting: a cross-sectional study. Psychosomatics. 2017;58(4):427-436. doi:10.1016/j.psym.20 17.02.008 
35. de Vroege L, Timmermans A, Kop WJ, van der Feltz-cornelis CM. Neurocognitive dysfunctioning and the impact of comorbid depression and anxiety in patients with somatic symptom and related disorders: a cross-sectional clinical study. Psychol Med. 2018;48 (11):1803-1813. doi:10.1017/S0033291717003300
36. de Vroege L, Koppenol I, Kop WJ, Riem MME, van der Feltzcornelis $\mathrm{CM}$. Neurocognitive functioning in patients with conversion disorder/functional neurological disorder. $J$ Neuropsychol. 2020;15 (1):69-87. doi:10.1111/jnp.12206

\section{Publish your work in this journal}

Neuropsychiatric Disease and Treatment is an international, peerreviewed journal of clinical therapeutics and pharmacology focusing on concise rapid reporting of clinical or pre-clinical studies on a range of neuropsychiatric and neurological disorders. This journal is indexed on PubMed Central, the 'PsycINFO' database and CAS, and is the official journal of The International Neuropsychiatric Association (INA). The manuscript management system is completely online and includes a very quick and fair peer-review system, which is all easy to use. Visit http://www.dovepress.com/testimonials.php to read real quotes from published authors.

Submit your manuscript here: https://www.dovepress.com/neuropsychiatric-disease-and-treatment-journal 